\title{
Unsupported off-label chemotherapy in metastatic colon cancer
}

\author{
Jonas A de Souza ${ }^{1,2^{*}}$, Blase Polite ${ }^{1,2,3}$, Monica Perkins ${ }^{4}$, Neal J Meropol ${ }^{5}$, Mark J Ratain ${ }^{1,2,6}$, Lee N Newcomer ${ }^{4}$ \\ and G Caleb Alexander ${ }^{7}$
}

\begin{abstract}
Background: Newer systemic therapies have the potential to decrease morbidity and mortality from metastatic colorectal cancer, yet such therapies are costly and have side effects. Little is known about their non-evidence-based use.
\end{abstract}

Methods: We conducted a retrospective cohort study using commercial insurance claims from UnitedHealthcare, and identified incident cases of metastatic colon cancer (mCC) from July 2007 through April 2010. We evaluated the use of three regimens with recommendations against their use in the National Comprehensive Cancer Center Network Guidelines, a commonly used standard of care: 1) bevacizumab beyond progression; 2) single agent capecitabine as a salvage therapy after failure on a fluoropyridimidine-containing regimen; 3) panitumumab or cetuximab after progression on a prior epidermal growth factor receptor antibody. We performed sensitivity analyses of key assumptions regarding cohort selection. Costs from a payer perspective were estimated using the average sales price for the entire duration and based on the number of claims.

Results: A total of 7642 patients with incident colon cancer were identified, of which 1041 (14\%) had mCC. Of those, 139 (13\%) potentially received at least one of the three unsupported off-label (UOL) therapies; capecitabine was administered to 121 patients and 49 (40\%) likely received it outside of clinical guidelines, at an estimated cost of $\$ 718,000$ for 218 claims. Thirty-eight patients received panitumumab and six patients (16\%) received it after being on cetuximab at least two months, at an estimated cost of $\$ 69,500$ for 19 claims. Bevacizumab was administered to 884 patients. Of those, 90 (10\%) patients received it outside of clinical guidelines, at an estimated costs of $\$ 1.34$ million for 636 claims.

Conclusions: In a large privately insured mCC cohort, a substantial number of patients potentially received UOL treatment. The economic costs and treatment toxicities of these therapies warrant increased efforts to stem their use in settings lacking sufficient scientific evidence.

Keywords: Colorectal cancer, Off-label, Evidence-based medicine, Physician practice patterns

\section{Background}

During the past two decades there have been many advances in the treatment of metastatic colorectal cancer. For half a century, 5-fluorouracil was the only treatment that demonstrated benefit and was thus used as a standard agent. Since the United States Food and Drug Administration (FDA) approved irinotecan, three

\footnotetext{
* Correspondence: jdesouza@medicine.bsd.uchicago.edu

${ }^{1}$ Section of Hematology/Oncology, The University of Chicago Medicine,

Chicago, IL, USA

${ }^{2}$ Comprehensive Cancer Center, The University of Chicago Medicine,

Chicago, IL, USA

Full list of author information is available at the end of the article
}

other cytotoxic agents and three biologic agents have been added to the armamentarium for the treatment of metastatic colorectal cancer. These therapeutic innovations have resulted in an increase in median survival from a baseline of 4 to 6 months (i.e., with supportive care alone), to approximately one year with 5 -fluorouracil, and to more than 20 months with the use of sequential chemotherapy including cytotoxic agents and biologics [1].

This scientific progress, associated with the increase in the number of available regimens, has come at considerable cost. The improvement in survival has been accompanied by an estimated 340-fold increase in the cost of drugs for

\section{Biomed Central}


treating colorectal cancer [2,3]. At the same time, although the FDA approves therapies for specific clinical, or labeled, indications, physicians are free to use licensed medicines for both FDA-approved and non-approved, or off-label, uses [4,5]. In oncology, an estimated $50 \%$ of prescribed therapies are used off-label [6]. While many of these uses have clinical evidence, and indeed, may reflect the standard of care, there are also off-label uses not supported by evidence, or unsupported off-label (UOL) use.

Given the increased number of regimens and available combinations for metastatic colorectal cancer, coupled with the terminal implications of a diagnosis of metastatic disease, we hypothesized UOL use of chemotherapy commonly occurs in this setting. Our aim was to describe how often UOL chemotherapy occurs in this cohort of patients with metastatic colon cancer.

\section{Methods}

\section{Data source}

We identified patients with colon cancer from the United Healthcare health insurance database, which represents over 70 million privately insured patients across all 50 states, the District of Columbia, and the U.S. Virgin Islands. The database, which captures care delivered by over 700,000 physicians and other health care providers within 5,200 hospitals, contains information on member demographics, and utilization, charges and allowed payments for covered services, including hospitalizations, outpatient procedures, physicians' office visits and outpatient prescriptions. Data available for each facility and professional service claim include dates of service and International Classification of Diseases (ICD-9-CM) diagnosis codes. Professional service claims also include Level I Healthcare Common Procedure Coding System (HCPCS) codes, also known as Current Procedural Terminology (CPT) procedure codes, as well as Level II HCPCS codes. We defined chemotherapies using codes from the CPT and Level II HCPCS. These claims also provide additional information about the medications dispensed including its National Drug Code (NDC) and date of dispensing. Institutional review board approval was obtained at The University of Chicago and at United Healthcare.

\section{Cohort derivation}

In our primary analyses, we identified patients with at least one colon cancer claim using the ICD-9-CM diagnosis code of colon cancer (153.x) in the top 3 diagnoses from January 1, 2007 to April 30, 2010. In order to restrict our cohort to incident cases, we used January 1, 2007 to June 30, 2007 as a 6-month "look-back or clean" period, and excluded individuals with a diagnosis of colon cancer prior to July 2007 [7]. We further excluded individual members with an insurance coverage gap greater than 6 months. In order to capture the full continuum of services and benefits provided, patients with coordination of benefits were also excluded. Additionally, a select set of members for whom UnitedHealthcare only provided administrative services were excluded because their data could not be used for research. Within the remaining cohort of patients with claims of colon cancer, we further excluded patients with claims indicating multiple primary malignancies. This criterion was applied to rule out patients with a malignancy other than colon cancer (e.g. lung cancer, breast cancer) who could potentially be treated with the same antineoplastic agents [8].

We used two methods to determine the metastatic colon cancer (mCC) cases. In our main analyses, we identified individuals as having $\mathrm{mCC}$ based on the receipt of at least one of four antineoplastic agents - irinotecan, bevacizumab, cetuximab and panitumumab - that are FDA-approved and recommended by available guidelines only for the metastatic disease setting. This method was chosen in order to avoid the inclusion of patients whose metastatic diagnosis represented a "rule-out" diagnosis (e.g. patients with Stage II colon cancer whose reason for a staging imaging study is "rule-out metastatic colon cancer" and are thus coded as metastatic patients) [9].

In a sensitivity analysis, we aimed to increase the positive predictive value and specificity of this method by decreasing the number of false positives (i.e. those labeled metastatic, when in fact they received one of these drugs in the adjuvant setting), by also requiring the presence of two diagnosis codes for metastatic disease (196.xx-199.xx) separated by 30 days or more [10,11]. As previously shown, requiring two metastatic codes to appear separated by at least 30 days, reduces the designation of metastases in initially non-metastatic patients to 5\% [9]. Using this approach, we considered the metastatic codes $196.2 x$ (intra-abdominal lymph nodes), 196.6 (pelvic nodes), 197.5x (large bowel) as locally advanced rather than metastatic disease [12]. Similarly, we omitted patients with the code 198.89 (secondary malignant neoplasm of unspecified site) because of its high false-positive rate [13].

\section{Definition of unsupported off-label use}

We used the National Comprehensive Cancer Network Clinical Practice Guidelines in order to identify regimens that lack sufficient scientific support and thus that are not recommended in metastatic colon cancer [14]. These guidelines are developed by recognized clinical experts, and consist of recommendations based on clinical trial results, expert evaluation, outcome analyses, and clinical experience [15]. As such, they are regarded as reflecting the standard of care by health plans, large employers and other payers, including the Centers for Medicare and Medicaid Services [16]. We focused on three UOL regimens for which recommendations have not been changed in these guidelines during the study period: (1) use of 
single agent capecitabine after progression on one or more fluoropyrimidine-based regimens such as FOLFOX or FOLFIRI [17], addressed as "shown to be ineffective" in the NCCN guidelines; (2) use of bevacizumab beyond cancer progression on a prior bevacizumab therapy, addressed as "insufficient data to support"; and (3) panitumumab or cetuximab after cancer progression on a prior epidermal growth factor receptor (EGFR) monoclonal antibody, addressed as "no data, nor is there a compelling rationale". In 2012, a Phase III clinical trial of bevacizumab in patients with metastatic colorectal cancer whose disease had worsened following first-line treatment has further showed overall survival benefit with the addition of this drug $[18,19]$. This manuscript analyzed data from 2007 to 2010 , prior to completion of this study, when evidence of its use was still scarce.

\section{Rules for defining therapies}

We examined the daily drug use for each patient in order to identify these therapies. We defined the sequential progression of therapies based on their temporal relationships using the dates of initiation and discontinuation provided in the administrative claims. We considered a treatment as a next line of therapy when an addition or substitution of chemotherapy or biologic agent was observed and the resulting drug regimen lasted $\geq 28$ days, the duration of at least one chemotherapy cycle [8].

Within the metastatic colon cancer claims, we identified patients with capecitabine claims. Single agent capecitabine was defined as its use not accompanied by any other chemotherapy drug both 14 days before and after capecitabine therapy. We were interested in patients who received single agent capecitabine after use of 5-fluorouracil or capecitabine in a prior line of therapy in the metastatic setting. Further, we identified patients who received bevacizumab in combination with either oxaliplatin or irinotecan. The switch of therapy regimens from bevacizumab and oxaliplatin to bevacizumab and irinotecan or vice versa was considered UOL use. Noteworthy, in order to better identify patients with disease progression on bevacizumab, we required that patients received a bevacizumab regimen for at least 28 days. By using this 28-day threshold, our goal was limit the number of patients included in the study who had their regimen changed due to acute toxicity rather than disease progression. The use of panitumumab after clinical failure on cetuximab, or cetuximab after failure on panitumumab, (ie, sequential use of an epidermal growth factor receptor [EGFR] inhibitor) was assessed by identifying claims that included both cetuximab and panitumumab. Observational evidence suggests that allergic reactions tend to occur within the first few weeks of administration of these agents when they do occur [20]. As such, in order to exclude those patients who were switched to the fully human monoclonal antibody panitumumab because of an allergic reaction to the murine chimeric monoclonal antibody cetuximab, only regimens that were administered for at least 2 months were included.

\section{Estimating costs of therapy}

We estimated the costs of therapy acquisition using the average sales price (ASP) for the entire duration of UOL therapies, a statutorily defined price based on the national average of manufacturers' sales prices from two earlier quarters plus a $6 \%$ margin [21]. Medicare part B and most private payers use some form of ASP-based payment. We further considered prices of doses of bevacizumab at $5 \mathrm{mg} / \mathrm{kg}$, panitumumab at $6 \mathrm{mg} / \mathrm{kg}$ and capecitabine at $1250 \mathrm{mg} / \mathrm{m} 2$ twice a day for 14 days for a $170 \mathrm{~cm}$, $70 \mathrm{~kg}$ man [22].

\section{Results}

\section{Cohort derivation}

From January 2007 through April 2010, we identified 38,161 patients with claims that included colon cancer. Of these, 22,564 cases (59\%) were considered incident cases, with no colon cancer claims within 6 months prior to July 2007. We further excluded 4959 patients with a coverage gap greater than 6 months, 5389 coordination of benefit patients, 2464 patients whose data use was restricted by UnitedHealthcare's administrative services only customers, and 2110 patients for having evidence of more than one malignancy. Our study cohort consisted of the remaining 7642 patients, reflecting those with full claims that included at least one first diagnosis code of colon cancer as the only malignancy from July 2007 to April 2010. See Figure 1 for a summary of the cohort derivation.

A total of 1041 (13.6\%) patients received at least one of the four chosen agents to determine the presence of metastatic disease (irinotecan, cetuximab, panitumumab and bevacizumab). Age range and geographical data are listed in Table 1 . In this privately insured population, $61 \%$ of patients were younger than 60 years.

\section{Use of UOL therapies}

From the cohort consisting of 1041 patients, capecitabine was administered to 121 patients and 49 (40\%) received it in an unsupported off-label context. Cetuximab was administered to 144 patients, while 38 patients received panitumumab. Notably, 6 patients (16\%) received panitumumab after being on cetuximab at least 2 months, which was defined as UOL use. There were no patients who received cetuximab after being on panitumumab. Finally, bevacizumab was administered to 884 patients. Of those, 90 (10\%) represented UOL use. In total, 139 (13.3\%) individual patients received at least one of the three UOL regimens. 


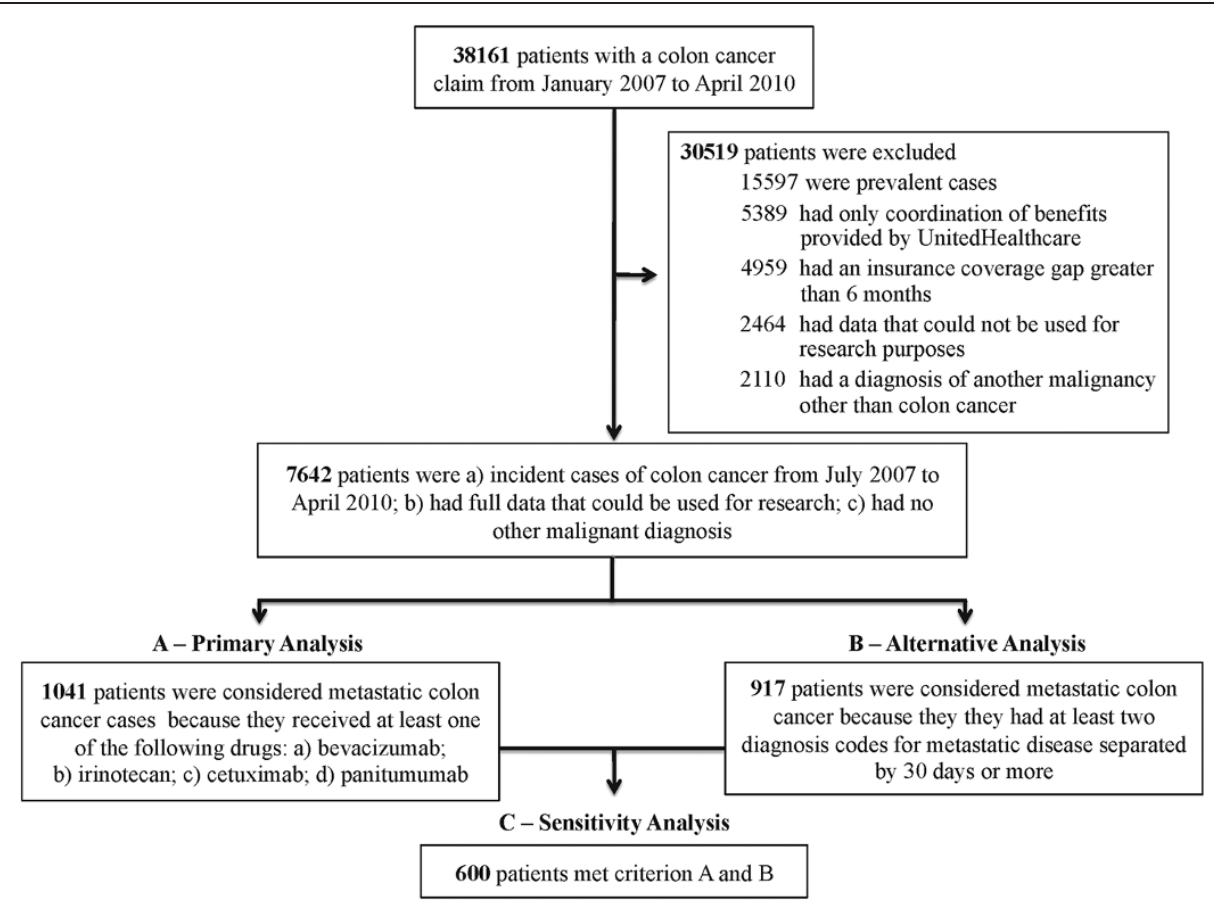

Figure 1 Algorithm for identifying patients with metastatic colon cancer.

\section{Sensitivity analyses on cohort derivation to increase specificity}

To further increase the specificity of our approach to identify metastatic cases, we identified 600 patients among those who had been considered metastatic cases based on administered drugs with at least two principal or secondary ICD-9-CM diagnosis codes indicating metastatic disease. In total, $116(19.3 \%)$ of these 600 patients received at least one

Table 1 Age and geographic regions of patients with metastatic colon cancer in the primary analysis $(N=1041)$

\begin{tabular}{lccc}
\hline & $\begin{array}{c}\text { All } \\
\text { subjects } \\
\mathbf{1 0 4 1}\end{array}$ & $\begin{array}{c}\text { Individuals receiving any of the } \\
\text { three unsupported off-label } \\
\text { therapies 139 (13\%) }\end{array}$ & $\begin{array}{c}\boldsymbol{p} \text { - } \\
\text { value }\end{array}$ \\
\hline Age & $277(27)$ & $44(32)$ & 0.014 \\
\hline 550 & $358(34)$ & $52(37)$ & \\
\hline $50-59$ & $242(23)$ & $34(24)$ & \\
\hline $60-69$ & $117(11)$ & $9(6)$ & \\
\hline$>70$ & $47(5)$ & 0 & \\
\hline Unknown & & $33(24)$ \\
\hline Region & $269(26)$ & $17(12)$ \\
\hline Midwest & $41(29)$ \\
\hline Northeast & $107(10)$ & $21(15)$ \\
\hline Southeast & $370(36)$ & $17(12)$ \\
\hline Southwest & $126(12)$ & $10(7)$ \\
\hline West & $96(9)$ & & \\
\hline Unknown & $72(7)$ &
\end{tabular}

of these three UOL regimens, as shown in Table 2. Among these patients, 38 received UOL capecitabine; 79 patients received bevacizumab beyond progression, and 5 patients received panitumumab after progressing on cetuximab.

\section{Number of claims and estimated treatment costs} associated with UOL regimens in the primary analysis

There were 636 claims for bevacizumab beyond progression. Considering the costs of drug acquisition using the average sales price (ASP) for the entire duration of UOL therapies, we thus estimated that $\$ 1.34$ million may have been used just to obtain that drug. Similarly, non-evidence based use of capecitabine was identified in 218 claims for a total cost of $\$ 718,000$. And nonevidence-based use of panitumumab was identified in 19 claims at an estimated cost of $\$ 69,500$. Within the 1041 metastatic colon cancer patients in the cohort, the estimated cost just to acquire the UOL drugs, which did not consider toxicities, ancillary support and infusion costs, was $\$ 2,127,500$.

\section{Discussion}

We found considerable utilization of expensive anticancer regimens in metastatic colon cancer without supporting evidence. We focused on treatments delivered after prior progression with the same or similar agents; more than one in eight patients received at least one regimen that is not considered standard of care. More importantly, the three assessed UOL uses have recommendations against their use 
Table 2 Age and geographic regions of patients with metastatic colon cancer in the sensitivity analysis $(\mathrm{N}=600)$

\begin{tabular}{lccc}
\hline & $\begin{array}{c}\text { All } \\
\text { subjects } \\
\mathbf{6 0 0}\end{array}$ & $\begin{array}{c}\text { Individuals receiving any of the } \\
\text { three unsupported off-label } \\
\text { therapies 116 (19\%) }\end{array}$ & $\begin{array}{c}\boldsymbol{p} \text { - } \\
\text { value }\end{array}$ \\
\hline Age & $163(27)$ & $37(32)$ & 0.153 \\
\hline$<50$ & $233(39)$ & $46(40)$ \\
\hline $50-59$ & $130(22)$ & $26(22)$ \\
\hline 60-69 & $62(10)$ & $7(6)$ \\
\hline Unknown & $12(2)$ & 0 \\
\hline Region & & $29(25)$ \\
\hline Midwest & $170(28)$ & $13(11)$ \\
\hline Northeast & $63(11)$ & $35(30)$ \\
\hline Southeast & $194(32)$ & $17(15)$ \\
\hline Southwest & $68(11)$ & $14(12)$ \\
\hline West & $56(9)$ & $8(7)$ \\
\hline Unknown & $49(8)$ &
\end{tabular}

in commonly adopted practice guidelines. With about 20,000 expected cases of colon cancer presenting with metastatic disease in 2011 [23], if we extrapolate an individual cost of $\$ 2043$ per metastatic case, a total of $\$ 40$ million may be related only to these $3 \mathrm{UOL}$ regimens in new metastatic cases. In addition to unnecessary exposure to toxicities and the economic burden of these therapies, UOL regimens may undermine the ability to enroll patients into clinical trials to rigorously assess such UOL clinical applications. For example, a clinical trial assessing bevacizumab beyond progression in $\mathrm{mCC}$, one of the therapies reported in this study, was closed due to poor accrual of patients in November 2010 [24].

Our findings are not the first regarding off-label use of prescription drugs. In a widely cited investigation, Radley et al [25] analyzed a nationally representative audit of office-based physicians and found that nearly one-fourth of all medication uses were for off-label purposes, and of these, nearly three-fourths lacked evidence of clinical efficacy. Despite this, less is known about the off-label use of oncology therapies, most of which are infusible drugs and not captured in office-based audits. In a prospective observational cohort study of previously untreated patients with metastatic colorectal cancer conducted to evaluate the safety and effectiveness of bevacizumab in combination with chemotherapy, approximately one-third of these patients received the nonevidence based bevacizumab beyond progression [26]. Similarly, an analysis of an integrated database of electronic medical records from 91 oncology practices indicated that among 1106 patients who received bevacizumabcontaining regimens for metastatic colon cancer, 280 (25\%) received it beyond progression in a subsequent line [8]. While this prior work considered all combinations of bevacizumab and other agents, we focused particularly on the use of bevacizumab and oxaliplatin after a prior use of bevacizumab and irinotecan and vice versa. To our knowledge, the UOL use of EGFR inhibitors and capecitabine has not been previously reported.

While our analysis was not designed for causal inference, there are several hypotheses regarding the UOL use that we document. First, patients with metastatic cancers and their treating physicians, when experiencing life-threatening illnesses with limited survival, may opt to receive and prescribe UOL regimens [27]. Many advocate that "even unapproved drugs often show early evidence of benefit reliable enough to use in desperately ill cancer patients" [28] or that "any glimmer of hope is better than none" [29]. Indeed, recent controversy surrounding the FDA's decision to withdraw approval of bevacizumab for metastatic breast cancer highlights the degree to which advocacy may conflict with health policy [30]. Second, current reimbursement methods do not provide financial incentives for the delivery of many aspects of care that are promoted by professional guidelines. Notably, in the 2011 National Practice Benchmark drugs and infusion revenue accounted for $73 \%$ of total revenue in 37 oncology practices, while evaluation and management activities were responsible for only $8 \%$ of this revenue [31]. Third, mastering the clinical evidence can be a difficult task, and even compendia that attempt to do so may contain inaccuracies and fail to reflect timely standards of care [32]. Finally, the impact of marketing strategies on the UOL use of chemotherapy drugs is unknown [33]. Bevacizumab, panitumumab and capecitabine are all FDA-approved and ethically promoted drugs in the metastatic colon cancer setting. However, they are often administered without sufficient evidence, as was also the case of bevacizumab prior to 2012 [19].

Our report has strengths and limitations. We believe it represents the first effort to systematically quantify the utilization and estimated drug costs of non-evidence based off-label regimens in metastatic colon cancer in a large private insurance claims database. However, the use of claims is subject to misclassification bias and thus may include some patients without metastatic colon cancer and exclude some patients with metastatic colon cancer. In order to minimize the effect of such bias, we based our cohort derivation on previously published methods, and further increased the positive predictive value of the algorithm by including chemotherapy agents used in this cohort of patients. Nevertheless, coding imprecisions may still be present, such as the coding of panitumumab as J9999 (not otherwise classified antineoplastic drug) prior to the creation of its HCPCS J code in 2008. In addition, our analyses are limited to the privately insured and 
predominantly younger population, with limited number of older individuals. One might then hypothesize that physician aggressiveness may increase in younger patients, and thus the increased likelihood of treatment with UOL in this population. Also, as with all claims-based analyses, our data provide limited insight regarding specific reasons that regimens were selected or discontinued. This is a particularly important limitation when assessing bevacizumab beyond progression, when, for example, a regimen containing oxaliplatin may have been switched to irinotecan due to neurotoxicity rather than disease progression. Similarly, switching from a 5 -fluorouracil based therapy (e.g. FOLFOX) to single agent capecitabine due to toxicity in a stop-and-go approach is an acceptable regimen, and it cannot be distinguished from salvage capecitabine after failure on 5-fluorouracil in claims analyses. In a prior analysis of patterns of care in metastatic colorectal cancer that included chart review for reasons for discontinuation, up to $18 \%$ of patients had their regimens changed due to issues related to toxicities and tolerability [34], and this should be seen as the main limitation of this study. Notably, in 2012 a Phase III clinical trial in patients with metastatic colorectal cancer whose disease had worsened following first-line treatment with bevacizumab plus standard chemotherapy (irinotecan or oxaliplatinbased) has showed overall survival benefit with the addition of this drug. This study analyzed data from 2007 to 2010, prior to evidence of this benefit, when the drug was still used as UOL $[18,19]$. Finally, our cost estimates are based on a payers' perspective and limited to the therapies examined, rather than other potential costs of treatment such as treatment complications, lost wages, work productivity or emotional and quality of life.

\section{Conclusions}

In view of the recent progress treating cancer, several newer therapies have been introduced at a rapid pace. In order to curtail costs in oncology while preserving efficacy and quality, a first step is identifying therapies with no or low value. This study provides us with hypothesis generating data that expensive UOL regimens are potentially being used in oncology, and further larger studies should further investigate causal relationships. In the policy arena, recent initiatives that incentivize providers to follow the best available evidence and reduce variations in care, such as the adoption of clinical pathways, as reported by Texas Oncology [35], or episode of care payments, piloted by UnitedHealthcare [36], hold the promise to decrease costs while improving outcomes, and should be further developed. Furthermore, discussions among several stakeholders about the appropriate use of practice guidelines, the level of evidence required for drug reimbursement, as well as the development of novel metrics to measure value in oncology become mandatory in our current health care debate. Utilization of unsupported and non-guideline based regimens in oncology, as well as factors associated with their use, may serve as targets for future health policy interventions.

\section{Competing interests}

Dr. Newcomer and Monica Perkins own stocks and are employed by UnitedHealthcare. Dr. Ratain served as consultant for Genentech, received research funds from Bristol-Myers Squibb, and provided expert testimony for Mylan, none related to this work. There are no other known financial conflicts of interest among any of the authors including but not limited to employment/affiliation, all grants or funding, honoraria, paid consultancies, expert testimony, stock ownership or options, and patents filed, received or pending.

\section{Authors' contributions}

JADS, BP, MJR and GCA designed the study. MP and LNN were involved in acquisition of the dataset. JADS and GCA accessed the data and performed the statistical analysis. All authors helped with the interpretation of the data. JADS, BP, MJR, NJM and GCA drafted the first version of the manuscript, and all authors contributed to subsequent versions and revised it critically for important intellectual content. All authors read and approved the final manuscript.

\section{Acknowledgements}

The authors gratefully acknowledge Shu Zhu for assistance with statistical analyses.

\section{Author details}

'Section of Hematology/Oncology, The University of Chicago Medicine, Chicago, IL, USA. ${ }^{2}$ Comprehensive Cancer Center, The University of Chicago Medicine, Chicago, IL, USA. ${ }^{3}$ Center for Interdisciplinary Health Disparities Research, The University of Chicago Medicine, 5841 South Maryland Avenue, MC 2115, Chicago, IL 60637-1470, USA. ${ }^{4}$ UnitedHealthcare, Edina, Minnesota, USA. ${ }^{5}$ Division of Hematology and Oncology, University Hospitals Case Medical Center Seidman Cancer Center, Case Western Reserve University, Case Comprehensive Cancer Center, Cleveland, Ohio, USA. ${ }^{6}$ Committee on Clinical Pharmacology and Pharmacogenomics and Center for Personalized Therapeutics, The University of Chicago, Chicago, IL, USA. 'Section of General Medicine and Center for Health and the Social Sciences, University of Chicago, Chicago, IL, USA.

Received: 2 May 2012 Accepted: 26 December 2012 Published: 29 December 2012

\section{References}

1. Catenacci DVT, Kozloff M, Kindler HL, Polite B: Personalized colon cancer care in 2010. Semin Oncol 2011, 38(2):284-308.

2. Schrag D: The price tag on progress-chemotherapy for colorectal cancer. N Engl J Med 2004, 351(4):317-319.

3. Wong Y, Meropol NJ, Speier W, Sargent D, Goldberg RM, Beck JR: Cost implications of new treatments for advanced colorectal cancer. Cancer 2009, 115:2081-2091.

4. Walton SM, Schumock GT, Lee KV, Alexander GC, Meltzer D, Stafford RS: Prioritizing future research on off-label prescribing: results of a quantitative evaluation. Pharmacotherapy 2008, 28(12):1443-1452.

5. Alexander GC, Stafford RS: Does comparative effectiveness have a comparative edge? JAMA 2009, 301(23):2488-2490.

6. American Society of Clinical Oncology: Reimbursement for Cancer Treatment: Coverage of Off-Label Drug Indications. J Clin Oncol 2006, 24(19):3206-3208. July 1, 2006.

7. Setoguchi S, Solomon D, Glynn R, Cook E, Levin R, Schneeweiss S: Agreement of diagnosis and its date for hematologic malignancies and solid tumors between medicare claims and cancer registry data. Canc Causes Contr 2007, 18(5):561-569.

8. Hess GP, Wang PF, Quach D, Barber B, Zhao Z: Systemic Therapy for Metastatic Colorectal Cancer: Patterns of Chemotherapy and Biologic Therapy Use in US Medical Oncology Practice. J Oncol Pharm Pract 2010, 6(6):301-307. 
9. Earle $C C$, Nattinger $A B$, Potosky $A L$, et al: Identifying cancer relapse using SEER-Medicare data. Med Care 2002, 40(8):75-81. Aug 2002.

10. Rao S, Kubisiak J, Gilden D: Cost of illness associated with metastatic breast cancer. Breast Cancer Res Treat 2004, 83(1):25-32.

11. Goldman DP, Jena AB, Lakdawalla DN, Malin JL, Malkin JD, Sun E: The value of specialty oncology drugs. Health Serv Res 2010, 45(1):115-132.

12. Cooper GS, Yuan Z, Stange KC, Dennis LK, Amini SB, Rimm AA: The sensitivity of Medicare claims data for case ascertainment of six common cancers. Med Care 1999, 37(5):436-444.

13. Lamont EB, Herndon JE 2nd, Weeks JC, et al: Measuring disease-free survival and cancer relapse using Medicare claims from CALGB breast cancer trial participants (companion to 9344). J Natl Cancer Inst. 2006, 98(18):1335-1338.

14. Benson AB, Arnoletti JP, Bekaii-Saab T, et al: Colon Cancer. J Natl Compr Canc Netw 2011, 9(11):1238-1290.

15. Winn RJ, McClure J: The NCCN Clinical Practice Guidelines in Oncology: A Primer for Users. J Natl Compr Canc Netw 2003, 1(1):5-13. March 1, 2003.

16. McGivney WT: Medicare Recognizes NCCN Compendium. J Natl Compr Canc Netw 2008, 6(7):635. August 1, 2008.

17. Hoff PM, Pazdur R, Lassere Y, et al: Phase II Study of Capecitabine in Patients With Fluorouracil-Resistant Metastatic Colorectal Carcinoma. J Clin Oncol 2004, 22(11):2078-2083.

18. A Study of Avastin (Bevacizumab) Plus Crossover Fluoropyrimidine-Based Chemotherapy in Patients With Metastatic Colorectal Cancer; 2012. Available at: http://clinicaltrials.gov/ct2/show/NCT00700102. Last accessed April 30th, 2012.

19. Avastin-based regimen extends survival when continued beyond initial treatment in patients with metastatic colorectal cancer. Investor Update, Basel, 26 Januiart 2012; 2012. Available at: http://www.roche.com/investors/ ir_update/inv-update-2012-01-26.htm. Last accessed April 30th, 2012.

20. Timoney J, Chung KY, Park V, Trocola R, Peake C, Saltz LB: Cetuximab use without chronic antihistamine premedication. J Clin Oncol 2006, 24(18):13521. June 20, 2006.

21. Danzon PM, Taylor E: Drug Pricing and Value in Oncology. Oncologist 2010, 15(1):24-31. March 1, 2010.

22. Centers for Medicare \& Medicaid Services: ASP Drug Pricing Files; 2011. Available at: http://www.cms.gov/apps/ama/license.asp?file=/ McrPartBDrugAvgSalesPrice/downloads/APR-2012-ASP-Pricing-File.zip. Last accessed on April 24th, 2012.

23. Jemal A, Bray F, Center MM, Ferlay J, Ward E, Forman D: Global cancer statistics. CA Cancer J Clin 2011, 61:69-90.

24. Phase III Trial of Irinotecan-Based Chemotherapy Plus Cetuximab (NSC-714692) or Bevacizumab (NSC-704865) as Second-Line Therapy for Patients with Metastatic Colorectal Cancer who have Progressed on Bevacizumab with either FOLFOX, OPTIMOX, or XELOX; 2012. http://www. swog.org/Nisitors/ViewProtocolDetails.asp?ProtocollD=2057 (Accessed 24 April 2012).

25. Radley DC, Finkelstein SN, Stafford RS: Off-label Prescribing Among Office-Based Physicians. Arch Intern Med 2006, 166(9):1021-1026.

26. Grothey A, Sugrue MM, Purdie DM, et al: Bevacizumab beyond first progression is associated with prolonged overall survival in metastatic colorectal cancer: results from a large observational cohort study (BRiTE). J Clin Oncol 2008, 26(33):5326-5334.

27. Levêque D: Off-label use of anticancer drugs. The Lancet Oncology 2008, 9:1102-1107.

28. DeVita VT: Off-label use of approved drugs. Nat Rev Clin Oncol 2009, 6:181.

29. Kaser E, Shaw J, Marven M, Swinburne L, Boyle F: Communication about high-cost drugs in oncology: the patient view. Ann Oncol 2010, 21:1910-1914

30. Robert HC: Bevacizumab: public opinion might trump science. The Lancet Oncology 2011, 12:730.

31. Barr TR, Towle EL: National Oncology Practice Benchmark, 2011 Report on 2010 Data. J Oncol Pharm Pract 2011, 7:67s-82s.

32. Abernethy AP, Raman G, Balk EM, et al: Systematic Review: Reliability of Compendia Methods for Off-Label Oncology Indications. Ann Intern Med 2009, 150:336-343.
33. Hampton T: Experts weigh in on promotion. Prescription of off-label drugs. JAMA 2007, 297:683-684.

34. Zafar SY, Marcello JE, Wheeler JL, et al: Longitudinal patterns of chemotherapy use in metastatic colorectal cancer. J Oncol Pharm Pract 2009, 5:228-233.

35. Hoverman JR, Cartwright TH, Patt DA, et al: Pathways, Outcomes, and costs in colon cancer: retrospective evaluations in two distinct databases. J Oncol Pharm Pract 2011, 7(3S):52s-59s.

36. Newcomer LN: Trying something new: episode payments for cancer therapy. J Oncol Pharm Pract 2011, 7(3S):60s-61s.

doi:10.1186/1472-6963-12-48

Cite this article as: de Souza et al:: Unsupported off-label chemotherapy in metastatic colon cancer. BMC Health Services Research 2012 12:481.

\section{Submit your next manuscript to BioMed Central and take full advantage of:}

- Convenient online submission

- Thorough peer review

- No space constraints or color figure charges

- Immediate publication on acceptance

- Inclusion in PubMed, CAS, Scopus and Google Scholar

- Research which is freely available for redistribution

Submit your manuscript at www.biomedcentral.com/submit
Ciomed Central 